\title{
Influence of Discrete Drop Locations on Film Thickness Uniformity in UV- Nanoimprint Lithography
}

\author{
Shrawan Singhal ${ }^{1}$ and S.V. Sreenivasan ${ }^{1,2}$ \\ ${ }^{1}$ NASCENT Center, The University of Texas at Austin \\ ${ }^{2}$ Canon Nanotechnologies, Inc., Austin, TX
}

\begin{abstract}
Multi-nozzle inkjetting is the material deposition approach for a variant of UVnanoimprint lithography called Jet-and-Flash Imprint Lithography (J-FIL). J-FIL has several advantages with respect to addressing pattern density variations in the template as well as achieving low material waste when compared with spin-coating. In this work, the influence of discrete drop placement in J-FIL on residual layer thickness uniformity has been investigated with the help of a novel nonlinear lubrication theory model, which takes into account the compliant nature of the template in the presence of a moving contact line. This work is directed at thin residual layers (sub-20 nm), where a correlation exists between minimum drop resolution, inkjet nozzle pitch and residual layer thickness uniformity. Numerical simulations reveal that the non-uniformity resulting from discrete drop placement can exceed desired process tolerances when low film thicknesses are desired with relatively larger drop volumes. The results have been verified experimentally to reveal the same trend. This work can be useful in determining the correlation of inkjet drop resolution with inkjet nozzle pitch to meet process tolerances in the JFIL process.
\end{abstract}

Keywords: Nanoimprint Lithography; Jet and Flash Imprint Lithography; Inkjetting; Lubrication Theory; Residual Layer Thickness Uniformity

\section{INTRODUCTION}

Nanoimprint lithography has been recognized as a successor to traditional photon-based lithography, particularly as critical dimensions continue to scale down to values that are far beyond the resolution limits of photon-based techniques [1]-[4]. Among the several flavors of 
nanoimprint lithography, Jet-and-Flash Imprint Lithography (J-FIL ${ }^{\mathrm{TM}}$ ) has been commercialized for semiconductor manufacturing [5]. It is a UV-based nanoimprint lithography technique relying on the use of a low-viscosity UV-curable monomer to fill the nanoscale resolution features on a master template. A typical J-FIL process flow has been shown in Figure 1. The preferred mode of depositing the UV-curable monomer is through a multi-nozzle piezoelectric inkjet [6]. J-FIL has been developed across three distinct process platforms: (i) wafer-based step and repeat imprinting [7], (ii) whole-wafer imprinting in a single step [8], and (iii) imprinting on flexible substrates in a roll-to-roll configuration [9]. The use of inkjetting is common across all these process platforms. It has several advantages, as well as several process challenges, that have been described next.
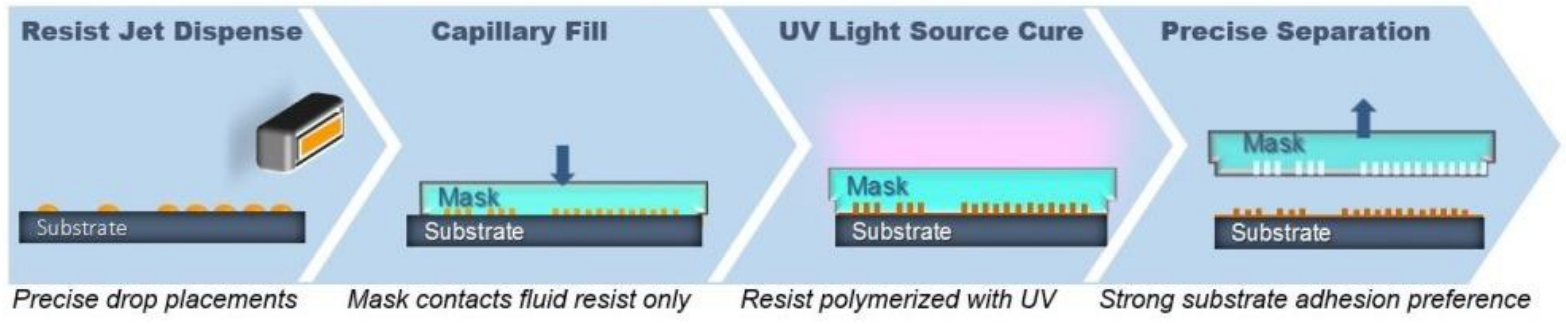

Figure 1: Process flow for Jet-and-Flash Imprint Lithography (J-FIL $\left.{ }^{\mathrm{TM}}\right)$. Courtesy: Canon Nanotechnologies

\subsection{Inkjetting}

Piezoelectric inkjets are widely used across several industries [10]. They lead to inherently low material waste, especially when compared to other material deposition processes [11]. Moreover, their ability to dispense 'drop-on-demand' allows placing drops where they are needed, leading to coarse two-dimensional 'patterning'. The primary disadvantage with using inkjets however, is that the material rheology challenges are substantially more difficult, especially when compared to other processes like spin-coating. The process of defect-free drop formation and dispense relies on a careful balance of piezoelectric actuation waveform parameters and inkjet geometry with the material rheology [12]. This is captured through plots of inkjettability that rely on bounds set by non-dimensional numbers such as the Ohnesorge number, Weber number and Capillary number [13]. However, these plots and non-dimensional 
numbers often do not address the issue of drop volume resolution, thereby making it difficult to achieve similar drop volumes across a range of different materials or inkjets. Thus, it can be imagined that when developing new UV-curable resist formulations, it becomes necessary to keep the material rheology consistent to enable inkjetting without significant deviation in drop formation and dispensing behavior. This can lead to a highly constrained material design problem, which can lead to sub-optimal functional characteristics. This is especially true for materials with extreme surface tension and viscosity, for which the only option might be to go with higher volume drops [14], [15].

In addition to this, the use of multiple nozzles on the same inkjet head creates additional challenges [16]. This configuration is desired from the standpoint of enabling higher throughput

in commercial manufacturing environments, as each nozzle is now able to dispense a drop simultaneously. However, the discrete distribution of the nozzles implies that drops are dispensed along discrete locations corresponding to multiples of the nozzle pitch. Ultimately, these discrete drops are merged to form a contiguous film under the action of the template in JFIL. If the drops are spaced too far apart, the drops can take too long to merge or lead to undesirable non-uniformity, which is discussed next in the context of the residual layer.

\subsection{Residual layer thickness uniformity}

The execution of any nanoimprint lithography process leads to the formation of a residual layer. This is a continuous film of resist that separates the patterned resist from the substrate, and needs to be etched before the resist pattern can be transferred to the substrate [17]. Thin residual layers are most desirable because they reduce the thickness that needs to be etched and hence, prevent any parasitic losses in feature critical dimensions. In addition to being thin, residual layers also need to have a substantially uniform thickness to minimize the same variations in their subsequent etching. While thin residual layers automatically lend themselves to better uniformity, their uniformity can be further enhanced by using thinner or more compliant templates [18]-[20]. Both thin residual layers and more compliant templates are desirable in 
preventing the corruption of the final film thickness with parasitic non-uniformity because of substrate nanotopography [21]. However, obtaining thinner residual layers, with the same inkjet drop volumes, requires that the discrete inkjetted drops be spaced farther apart. As mentioned above, this can potentially lead to issues with undesirable non-uniformity during the formation of the contiguous film.

\subsection{Motivation}

Earlier work on the fluid mechanics of the J-FIL and related processes has mostly focused on the fluid behavior in the presence of a rigid template [22]-[27]. It is important to model the compliance of the template in order to capture the effect of varying distances between neighboring drops. In this context, the behavior of individual drops and moving contact lines has also been modeled in the literature, but these models do not adequately capture the spreading of a drop, or the movement of a contact line bound by two plates where one is compliant [28]-[33]. The goal behind this work is to investigate the process of formation of the contiguous fluid film starting from discrete inkjetted drops with the help of a semi-analytical model. The motivation behind this investigation is to understand the influence of discrete drop locations on the nonuniformity of the contiguous film with different drop volumes. Different template thicknesses have also been considered, as changing template thickness is akin to changing template stiffness or compliance. As mentioned earlier, material constraints may not allow the dispensing of small volumes for novel resist formulations. In such situations, the only recourse might be to dispense higher volumes, which can have implications for maintaining thin and uniform residual layers as larger volume drops have to be placed farther apart. Hence, this modeling exercise helps in deducing an optimum drop placement pitch that keeps the non-uniformity of the liquid film prior

to merging within desirable process tolerances. Any non-uniformity resulting from the drop spreading and merging process itself can potentially disrupt the uniformity of the final contiguous film formed after all the drops have merged, leading to undesirable process 
variations. The model developed in this work has been validated experimentally with good agreement.

\section{Modeling}

In previous work, a baseline model had been developed to understand the mechanics after the drops had merged to form a contiguous "macroscopic" film [18]. In this work, the same model has been extended to potentially capture the "mesoscopic" process of individual drop spreading prior to merging. In the J-FIL process, the imprint monomer is highly wetting and forms a low contact angle on both the template and the wafer. Hence, the radius of a drop $(\sim 100$ um) tends to be much larger than the height ( $<1$ micron), validating the use of lubrication approximation to model thin film flow [22]. The basic physics is given by thin film fluid flow confined by one rigid and one elastic substrate [18]. In this case, the wafer is assumed to be rigid, as it is held against a vacuum chuck. However, the template is assumed to be compliant as it is only chucked at the edges. To facilitate the modeling process, the template is assumed to be featureless and thus consists of an unpatterned plate of fused silica. The spreading of individual drops can be construed as the driving of contact lines by the wetting characteristics of the fluid as well as the coercing of the template. It must be noted that this work specifically relates to the spreading of individual drops prior to merging. The process of drop merging itself cannot be captured by this model, as it violates the basic lubrication assumption of negligible pressure gradients in the thickness direction [34], [35].

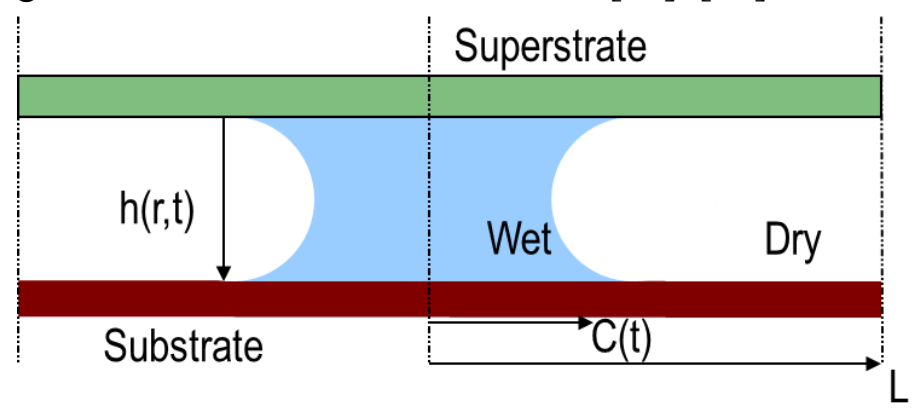

Figure 2: Geometry of the system under investigation for the mesoscopic model. A single drop of liquid is confined between the top compliant template and the bottom rigid substrate. 


\subsection{Problem formulation}

The system is modeled in axisymmetric radial co-ordinates as a periodic array of droplets at a constant pitch, which is given by a multiple of the inkjet nozzle pitch. The imprint monomer is assumed to be a wetting liquid (small contact angle on both substrates) upon which a top surface with an initial flat profile is imposed as shown in Figure. The topography on both the template and substrate is assumed to be zero and the system is modeled as being symmetric about the half-pitch of a given period containing a single droplet. Thus, with the given geometry, the given spatial half period consists of two regions, one containing the liquid (wet region 1) and the other without any fluid (dry region 2), with a meniscus or contact line at the boundary. It is further assumed that the shape of the contact line does not change from its static profile as the drop spreads. In other words, the contact angles of the fluid on both, the template and substrate, do not vary with time.

The flow of a thin film confined by an elastic plate is given as a coupling between the thin film lubrication equation and the thin plate bending equation [18]. This results in a sixth order unsteady partial differential equation given as:

$$
\frac{\partial h}{\partial t}=\frac{D}{12 \mu} \vec{\nabla} \cdot\left(h^{3} \vec{\nabla} \nabla^{4} h\right) \quad \text { Equation } 1
$$

In this equation $1, h$ and $t$ give the local film thickness and time, respectively. The viscosity of the fluid is given by $\mu$, and the bending stiffness of the elastic plate is given by

$$
D=\frac{E b^{3}}{12\left(1-v^{2}\right)}, \quad \text { Equation } 2 .
$$

In equation 2, $E, b$, and $v$ give the Young's modulus, thickness and Poisson's ratio of the template. Region 1 (labeled as wet in Figure 2.4) is governed by this sixth-order unsteady evolution equation, while it is assumed that region 2 (labeled as dry in Figure ) is only acted upon by a pressure field with no fluid. Thus, the deformation of the template, and hence, the local film thickness in this region is given by a fourth-order ordinary differential equation [36]. It is further assumed that there is negligible pressure applied on the template, a scenario which is consistent with the J-FIL process where only a slight pressure is applied to the template to initiate contact at the center of the substrate. The pressure is then relaxed upon initiation of 
spreading. Along with the local film thickness $h(r, t)$, the position of the contact line, $C(t)$, also evolves with time under a constraint of volume conservation, assuming no evaporation. Given the order of the governing equations, one initial condition for the film thickness, eight boundary conditions in $h$ for thin plate bending (four each for the two regions) and two more in $p$ for the coupled evolution equation are required to adequately constrain the system. Since the edges of the domain are effectively lines of symmetry, the odd derivatives are zero. This yields the following boundary conditions:

For $r=0: \frac{\partial h_{1}}{\partial r}=\frac{\partial \nabla^{2} h_{1}}{\partial r}=\frac{\partial p}{\partial r}=0 \quad$ Equation 3

For $r=L: \frac{\partial h_{2}}{\partial r}=\frac{\partial \nabla^{2} h_{2}}{\partial r}=0 \quad$ Equation 4

The subscript on $h$ in equations 3 and 4 denotes the region in which the boundary condition holds. The fifth derivative of $h_{1}$ is the same as the pressure gradient. Since there is no fluid at $r=L$, a similar condition does not exist for the right symmetry edge. This accounts for five boundary conditions. The remaining five are obtained by conditions at the meniscus, i.e., $r=C$ in Figure . Four of these reflect continuity in the film thickness, slope (first derivative), bending moment (second derivative) and shear force (third derivative) at the contact line going from region 1 to region 2 as follows:

For $r=C(t): \quad h_{1}=h_{2}, \frac{\partial h_{1}}{\partial r}=\frac{\partial h_{2}}{\partial r}, \nabla^{2} h_{1}=\nabla^{2} h_{2}, \frac{\partial \nabla^{2} h_{1}}{\partial r}=\frac{\partial \nabla^{2} h_{2}}{\partial r} \quad$ Equation 5

The last condition is obtained from the Young-Laplace equation which relates the pressure differential across a meniscus to the curvature and surface energy, $\gamma$, of the interface. With a wetting liquid on both the substrate and the superstrate, this yields the following nonlinear condition:

For $r=C(t): p=-\gamma f(\phi) / h_{1} \quad$ Equation 6

In the above equation $6, \gamma$ is the surface tension of the imprint monomer and $f(\phi)$ is a function of the contact angles of the liquid on the two surfaces, given by $\phi_{1}$ and $\phi_{2}$, where typically, $f(\phi)=\cos \left(\phi_{1}\right)+\cos \left(\phi_{2}\right)$. Since region 2 is not acted upon by any pressure, the profile of the template in region 2 is given by the homogeneous solution to the following 
equation: $\nabla^{4} h_{2}=0$. For axisymmetric geometry, this homogeneous equation yields a solution of the form:

$h_{2}=b_{0}+b_{1} r^{2}+b_{2} \log (r)+b_{3} r^{2} \log (r) \quad$ Equation 7

For region 1, the formulation of the thickness profile at any given instant of time is more complicated as the pressure field is unknown. Now, it can be observed that with a constant pressure, $p_{0}$, applied to the template in region 1 , the thickness profile would essentially be given as:

$h_{1}=a_{0}+a_{1} r^{2}+a_{2} \log (r)+a_{3} r^{2} \log (r)+\frac{p_{0} r^{4}}{64 D} \quad$ Equation 8

Thus, it can be assumed that the thickness is then given by the above equation 8 , but superposed by an additional unknown function that represents the variation in the film thickness caused by a spatially varying pressure field. This unknown function is expressed as an eigenfunction expansion with Bessel functions, $J_{0}$. The series expansion is truncated to a maximum of $N$ terms. The exact form of the eigenvalues, $k_{n}$, as well as the coefficients, $H_{n}$, of the series solution is not known yet. Hence, this yields the following form of the solution for $h_{1}$ : $h_{1}=a_{0}+a_{1} r^{2}+a_{2} \log (r)+a_{3} r^{2} \log (r)+\frac{p_{0} r^{4}}{64 D}+\sum_{n=1}^{N} H_{n}(t) J_{0}\left(k_{n} r\right) \quad$ Equation 9

The equations 8 and 9 are then solved for an initial condition of a zero pressure flat superstrate profile using spectral methods [18], [37]. This approach helps reduce the time evolution partial differential equation into a system of ordinary differential equations in time, that have been solved using the ode15s solver in MATLAB, which is a $1^{\text {st }}$ order explicit RungeKutta solver with variable time-stepping optimized for stiff systems. The simulation is stopped when $C \rightarrow L$, i.e., when two neighboring drops are close to merging, or when the film thickness becomes 0 , i.e., the superstrate comes in contact with the substrate, which is a singularity for thin film evolution. At every instance of time, strict volume conservation is also enforced, which gives the instantaneous value of the contact line position, or C. Values of some important parameters have been given in Table 1. 
Table 1: Values of important parameters and variables in the model.

\begin{tabular}{|l|l|l|l|l|l|l|l|l|}
\hline $\begin{array}{l}\text { Drop } \\
\text { volume }\end{array}$ & $\begin{array}{l}\text { Drop } \\
\text { pitch }(2 \mathrm{~L})\end{array}$ & $\begin{array}{l}\text { Monomer } \\
\text { viscosity } \\
(\mu)\end{array}$ & $\begin{array}{l}\text { Monomer } \\
\text { surface } \\
\text { tension } \\
(\gamma)\end{array}$ & $\begin{array}{l}\text { Contact } \\
\text { angle on } \\
\text { template } \\
\left(\phi_{1}\right)\end{array}$ & $\begin{array}{l}\text { Contact } \\
\text { angle on } \\
\text { wafer }\left(\phi_{2}\right)\end{array}$ & $\begin{array}{l}\text { Template } \\
\text { Young's } \\
\text { modulus } \\
(\mathrm{E})\end{array}$ & $\begin{array}{l}\text { Template } \\
\text { Poisson's } \\
\text { ratio }(v)\end{array}$ & $\begin{array}{l}\text { Template } \\
\text { thickness } \\
(\mathrm{b})\end{array}$ \\
\hline $\begin{array}{l}\text { Multiple } \\
\text { of } 6 \\
\text { picoliter }^{\ddagger}\end{array}$ & $\begin{array}{l}\text { Multiple } \\
\text { of } 84.5 \\
\mathrm{um}^{\S}\end{array}$ & $10 \mathrm{cp}$ & $30 \mathrm{mN} / \mathrm{m}$ & $\begin{array}{l}25 \\
\text { degrees }\end{array}$ & 9 degrees & $73 \mathrm{GPa}$ & 0.17 & $\begin{array}{l}0.7 \\
0.25 \mathrm{~mm}\end{array}$ \\
\hline
\end{tabular}

For the initial condition, the initial spread of the drop is calculated by assuming that the dispensed drop takes on the shape of a spherical cap with an approximate contact angle of 9 degrees on the substrate and 25 degrees on the template, as given in Table 1. These contact angles are representative of monomer resist formulations Monomat ${ }^{\mathrm{TM}}$ on Transpin $^{\mathrm{TM}}$ substrates coated with adhesion layer*. The objective for the simulation is to investigate the influence of varying template compliance along with varying drop pitch in multiples of the drop pitch.

\subsection{Numerical Verification}

Because of the presence of a nonlinear governing equation and nonlinear boundary condition, verification of the numerical code was undertaken by increasing the no. of terms in the series expansion and checking for convergence. For this purpose, the drops have been assumed to be dispensed at a pitch of 10 times the nozzle pitch, which is 84.5 um apart. This case represents an extreme, as with increasing distance between neighboring drops, there is greater chance of the model running into numerical singularities. The template thickness is assumed to be 700 microns. In the convergence analysis, three key metrics have been tracked: (i) mean film thickness, which gives a measure of volume conservation, (ii) \% non-uniformity or the ratio of standard deviation with mean film thickness, which is a desired output metric, and (iii) time taken for neighboring drops to merge, which is related to the throughput of the process. If the values of these three metrics change substantially with increasing number of terms, it would

\footnotetext{
${ }^{*}$ The monomer resist formulation used in this work is a proprietary material, Monomat ${ }^{\mathrm{TM}}$, obtained from Molecular Imprints, Inc. The adhesion layer is also a proprietary material, Transpin ${ }^{\mathrm{TM}}$, obtained from Molecular Imprints, Inc.

${ }^{\dagger}$ The template is typically made of fused silica.

* The drop volume is used to calculate the initial condition, i.e., the initial position, $\mathrm{C}$, and the initial height of the drop.

$\S$ The inkjet nozzle pitch is 84.5 um. Hence, the full drop pitch, i.e., 2L, corresponds to a multiple of 84.5 um.
} 
imply that the solution is not converging. Results have been summarized in Table 2 below and show that there is practically no change in the values of these metrics with increasing number of terms in the eigenfunction expansion. Hence, the solution is assumed to be converged and the code is assumed to be verified for $\mathrm{N}=30$.

Table 2: Results from convergence tests for numerical code verification

\begin{tabular}{|l|l|l|l|}
\hline $\mathrm{N}$ & Mean $(\mathrm{nm})$ & \%non-uniformity & Merge time $(\mathrm{s})$ \\
\hline 30 & 6.9814 & 5.1733 & 3.3739 \\
\hline 50 & 6.9815 & 5.1734 & 3.3738 \\
\hline 70 & 6.9811 & 5.1733 & 3.3742 \\
\hline
\end{tabular}

\subsection{Simulation Results}

After verifying the numerical results, simulations were conducted with the same drop volume of 6 picoliter dispensed at increasing pitch and merged with templates having thickness $700 \mathrm{um}$ and $250 \mathrm{um}$. For this purpose, too, the same three metrics of mean film thickness, \% nonuniformity and merge time have been tracked. The results are shown in Figure .
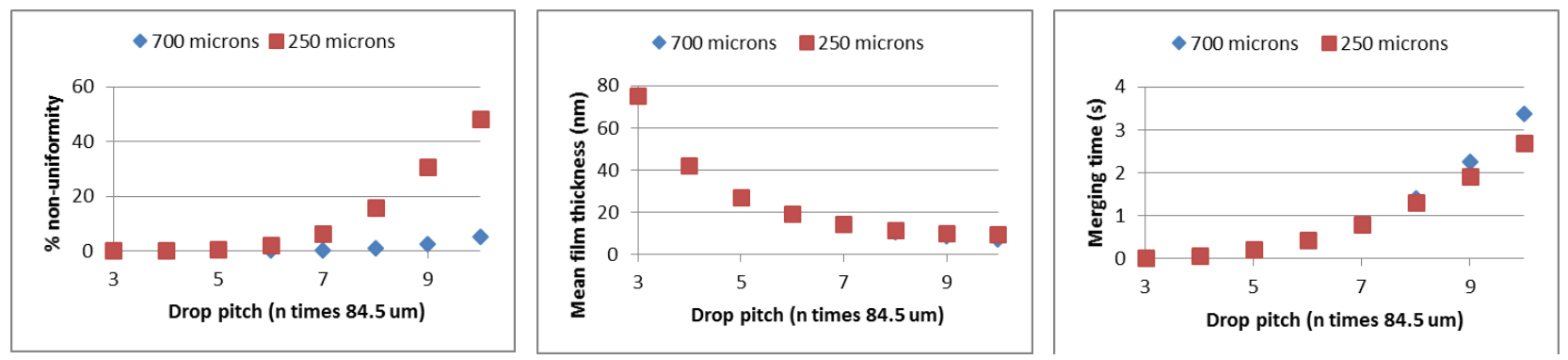

Figure 3: Simulation results (from left to right) showing variation of \% non-uniformity, mean film thickness and merging time with increasing drop pitch and changing template thickness.

It can be seen that drops spaced farther apart result in thinner films and take longer to merge, which is expected for both template thicknesses. However, the \% non-uniformity is substantially different for thin and thick templates at sub-20nm films. The thin template results in higher non-uniformity. This can be attributed to the fact that the work done to bend a thin plate is less than the same for a thick plate. With reducing film thickness, the capillary pressure is also increased, as the pressure is inversely proportional to film thicknes per equation 6 . This is 
responsible for bending the templates. Hence, a significant departure from the initial flat profile is seen as mean film thickness and template thickness, both are reduced, and becomes especially pronounced for sub-20nm thick films. However, it is desirable to move to lower template thickness or compliance when addressing full wafer nanotopography [18]. This implies that there is an inherent trade-off in the compliance requirements of the template. It should not be so compliant that it leads to substantial non-uniformity during drop merging, and at the same time, it should not be so rigid that it leads to parasitic non-uniformity because of lack of wafer flatness over large scales. The merging times, which is a key driver for process throughput, are similar for both templates, except at very thin films. There, the thicker template takes longer to force the drops to merge. This can again be attributed to the fact that thicker template does not bend as much and as such, results in a flatter profile compared to the thinner template. At the moving contact line, this translates to a larger film thickness and hence, a lower capillary pressure that drives the drop to merge with its neighboring drop.

\section{EXPERIMENTAL VALIDATION}

In the simulation results shown above, the drop volumes used were 6 picoliters, and as was discussed previously, it may not always be feasible to get the same drop volumes with different material formulations. Hence, as a preliminary test to understand process behavior at large drop volumes spaced farther apart, experiments have been conducted with the same UVcurable monomer for different drop volumes to achieve the same film thicknesses of $\sim 70 \mathrm{~nm}$. This meant that larger drops had to be spaced farther apart. Since the inkjet drop volume resolution is 6 picoliters, multiple drop dispense steps have been conducted prior to the coercing action by the template. The experimental results have been compared against simulations with the same parameters.

\subsection{Experimental Methods}

The experiments have been conducted on an Imprio-1100 whole wafer imprint tool from Molecular Imprints, Inc. The UV-curable resist used is a proprietary material from the same 
company, sold under the trade name of Monomat. The substrates used were Si wafers having a diameter of 3 inches and the templates were fused silica wafers having a diameter of $150 \mathrm{~mm}$ with no patterns. Prior to performing the experiments, the wafer surface was pre-treated with a thin adhesion layer coating. This improved the wetting properties of the Si wafer and allowed a contact angle of approximately 9 degrees for the liquid resist on the Si wafer. The Imprio-1100 tool is equipped with a multi-nozzle inkjet printhead, with individual nozzles spaced $84.5 \mathrm{um}$ from each other. The inkjet is tuned to dispense the liquid resist in steps of 6 picoliter drops. The imprint was conducted across a circular area of $65 \mathrm{~mm}$ diameter on the Si wafers. The final cured resist film thickness was measured on a Metrosol VUV system on 49 points over the wafer. The $\%$ non-uniformity has been calculated as the ratio between standard deviation and the mean film thickness.

\subsection{Experimental Results}

Qualitative validation of the predictions of the mesoscopic model has been conducted by using drop patterns of different pitch and volume to obtain a mean film thickness of $\sim 70 \mathrm{~nm}$ with a 250 um thick template. In order to minimize corruption of the final film thickness, the liquid film was cured immediately after all the drops were merged to form a contiguous fluid film. The experimentally measured non-uniformity has been compared against the mesoscopic model prediction in Figure. The experimentally measured non-uniformity has been reduced to account for the maximum non-uniformity of the adhesion layer coating, which is approximately $0.6 \mathrm{~nm}$, as this non-uniformity has not been captured in the model. As can be seen, the trend of increasing non-uniformity with increasing drop pitch is reflected in both the model and experiment. Thus, it can be said that the model qualitatively agrees with the experimental data.

There are several sources of variation in the experiments when compared with the model. The model assumes that both substrate and template are perfectly flat. In reality, this is not true and any parasitic topography on these two surfaces can corrupt the final film thickness uniformity [18]. In the actual experiment, the drops do not merge all at once, whereas this is the 
implicit assumption in the model. Other secondary sources of error include the inkjet drop volume accuracy, which has been measured as nearly $10 \%(1 \sigma)$, and the inkjet drop placement accuracy which is approximately $3 \mu \mathrm{m}(1 \sigma)$. While these factors lead to a difference in the expected values, overall, the trend of increasing non-uniformity with increasing drop pitch for the same mean film thickness has been captured. This can be used to inform inkjet drop volume constraints based on the available inkjet nozzle pitch.

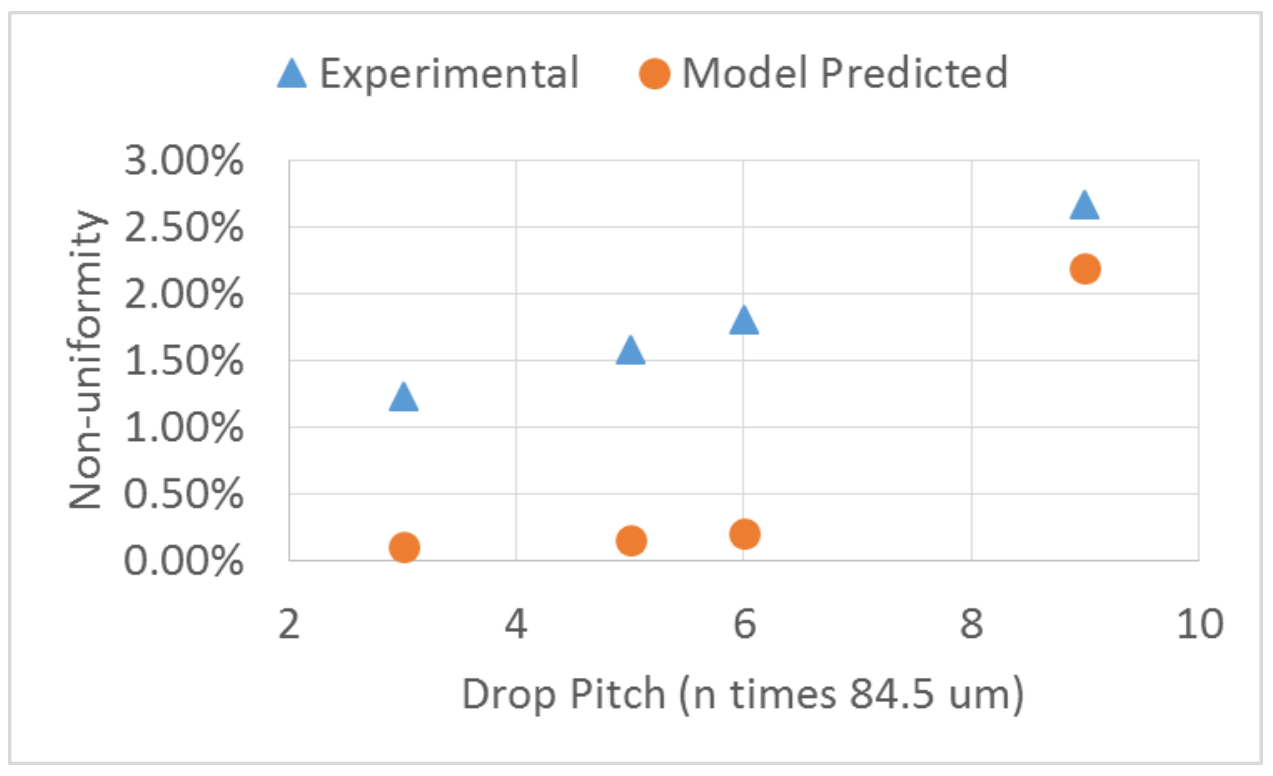

Figure 4: Comparison of experimental result and model prediction for drops of different volume and pitch for achieving a similar mean film thickness with a 250 um thick template.

\section{Conclusions}

In this article, it has been shown that the use of multi-nozzle inkjets to dispense drops in J-FIL can lead to undesirable non-uniformity, particularly at thin residual layers (sub-20nm). For example, from Figure, it can be seen that with a 250um thick template, a 6 picoliter drop, if dispensed more than 7 times the nozzle pitch apart (as needed for sub-20nm films), can lead to $>5 \%$ non-uniformity even before the drops have merged to form a contiguous film. Experimental evidence suggests that the non-uniformity tends to be higher than the model prediction, thereby indicating that it may be very difficult to maintain sub-5\% non-uniformity in situations where sub-20nm thin films are desired at 6 picoliter volumes. This problem gets worse if the inkjet can 
only dispense larger volume drops. If residual layer thickness uniformity becomes a challenge, it is desirable to thus, move towards smaller drops and inkjets with nozzles spaced closer together. Such an inkjet configuration can alleviate problems associated with undesirable non-uniformity both before and after merging of inkjetted drops. The experimentally validated model developed in this article can thus be used as a baseline for optimizing the inkjet drop volume resolution for a given inkjet multi-nozzle configuration to enable control of residual layer thickness uniformity. It should also be pointed out here that the work done in this article has been validated for a blank, featureless template. A typical J-FIL process template will have multiple patterns, often of varying density. Hence, the film thickness values and the corresponding drop pitch described in this article will not be commensurate with the residual layer thickness with patterned templates. However, the model can still be used in this scenario by reducing the initial drop volume by the volume occupied by the pattern. In this manner, only the volume of the drop responsible for the residual layer thickness will be modeled and would thus be decoupled from the presence of a pattern.

In conclusion, this article presents three primary contributions to the state-of-the-art in JFIL. The first contribution includes the formulation and experimental validation of a novel extension of a nonlinear lubrication theory model by incorporating compliant templates and moving contact lines to capture the influence of the spreading of discrete drops on the J-FIL process. Secondly, the simulations presented in this article reveal important trade-offs in inkjet system design and associated process control. This has implications in material design and optimization for inkjettability, as well as control of residual layer thickness for maintaining uniformity of the features after etching. Finally, this article also presents an important trade-off in the choice of compliance of the template. It has to be carefully chosen to address full wafer nanotopography, which can be as high as $1 \mu \mathrm{m}$, while also minimizing non-uniformity caused during the drop merging process. 


\section{ACKNOWLEDGEMENTS}

This work has been sponsored by the National Science Foundation under the Scalable Nanomanufacturing Program (ECCS 1120823).

\section{REFERENCES}

[1] S. V. Sreenivasan, "Nanoscale Manufacturing Enabled by Imprint Lithography," MRS Bulletin, vol. 33, no. 09, pp. 854-863, 2008.

[2] S. Chou, P. Krauss, and P. Renstrom, "Nanoimprint lithography," JOURNAL OF VACUUM SCIENCE \& TECHNOLOGY B, vol. 14, no. 6, pp. 4129-4133, Dec. 1996.

[3] Y. Xia and G. M. Whitesides, "Soft lithography," Annual Review of Materials Science, vol. 28, pp. 153-184, 1998.

[4] M. Bender, M. Otto, B. Hadam, B. Vratzov, B. Spangenberg, and H. Kurz, "Fabrication of nanostructures using a UV-based imprint technique," Microelectronic Engineering, vol. 53, no. 1-4, pp. 233-236, Jun. 2000.

[5] S. V. Sreenivasan, B. J. Choi, P. D. Schumaker, and F. Xu, "Status Of UV Imprint Lithography For Nanoscale Manufacturing," in Comprehensive Nanoscience And Technology, vol. 4, Oxford Academic Press, 2011, pp. 83-116.

[6] Z. Ye, R. Ramos, C. Brooks, L. Simpson, J. Fretwell, S. Carden, P. Hellebrekers, D. LaBrake, D. J. Resnick, and S. V. Sreenivasan, "High-density patterned media fabrication using jet and flash imprint lithography," in SPIE Advanced Lithography, 2011, p. 79700L79700L.

[7] S. V. Sreenivasan, B. J. Choi, N. E. Schumaker, R. D. Voisin, M. P. C. Watts, and M. J. Meissl, "Step and repeat imprint lithography processes," 7727453, 01-Jun-2010.

[8] D. Lentz, G. Doyle, M. Miller, G. Schmidt, M. Ganapathisubramaniam, X. Lu, D. J. Resnick, and D. LaBrake, "Whole wafer imprint patterning using step and flash imprint lithography: a manufacturing solution for sub-100-nm patterning," in Proceedings of SPIE Advanced Lithography, Emerging Lithographic Technologies XI, 2007, vol. 6517, p. 65172F.

[9] S. Ahn, M. Ganapathisubramanian, M. Miller, J. Yang, J. Choi, F. Xu, D. J. Resnick, and S. V. Sreenivasan, "Roll-to-roll nanopatterning using jet and flash imprint lithography," 2012, vol. 8323, p. 83231L-83231L-7.

[10] M. Singh, H. M. Haverinen, P. Dhagat, and G. E. Jabbour, "Inkjet Printing-Process and Its Applications," Advanced Materials, vol. 22, no. 6, pp. 673-685, Feb. 2010.

[11] F. C. Krebs, "Fabrication and processing of polymer solar cells: a review of printing and coating techniques," Solar Energy Materials and Solar Cells, vol. 93, no. 4, pp. 394-412, 2009.

[12] B. Derby, "Inkjet Printing of Functional and Structural Materials: Fluid Property Requirements, Feature Stability, and Resolution," Annual Review of Materials Research, vol. 40, no. 1, pp. 395-414, 2010.

[13] H. C. Nallan, J. A. Sadie, R. Kitsomboonloha, S. K. Volkman, and V. Subramanian, "Systematic Design of Jettable Nanoparticle-Based Inkjet Inks: Rheology, Acoustics, and Jettability," Langmuir, vol. 30, no. 44, pp. 13470-13477, Nov. 2014. 
[14] L. C. Glangchai, M. Caldorera-Moore, L. Shi, and K. Roy, "Nanoimprint lithography based fabrication of shape-specific, enzymatically-triggered smart nanoparticles," Journal of Controlled Release, vol. 125, no. 3, pp. 263-272, Feb. 2008.

[15] V. Singh, R. Agarwal, P. Jurney, K. Marshall, K. Roy, L. Shi, and S. V. Sreenivasan, "Scalable Fabrication of Low Elastic Modulus Polymeric Nanocarriers With Controlled Shapes for Diagnostics and Drug Delivery," J. Micro Nano-Manuf, vol. 3, no. 1, pp. 011002-011002, Mar. 2015.

[16] I. M. Hutchings and G. D. Martin, Inkjet Technology for Digital Fabrication. John Wiley \& Sons, 2012.

[17] S. Singhal, R. Attota, and S. V. Sreenivasan, "Residual layer thickness control and metrology in jet and flash imprint lithography," in SPIE Advanced Lithography, 2012, pp. 832434-832434.

[18] S. Singhal, M. J. Meissl, R. T. Bonnecaze, and S. V. Sreenivasan, "Inkjet-based deposition of polymer thin films enabled by a lubrication model incorporating nano-scale parasitics," Physics of Fluids, vol. 25, pp. 092002-092002, Sep. 2013.

[19] S. Singhal and S. V. Sreenivasan, "Nano-scale mechanics of front-side contamination in photonic device fabrication using imprint lithography," presented at the 22nd Annual Meeting of the American Society for Precision Engineering, ASPE 2007, 2007.

[20] R. Ji, M. Hornung, M. A. Verschuuren, R. van de Laar, J. van Eekelen, U. Plachetka, M. Moeller, and C. Moormann, "UV enhanced substrate conformal imprint lithography (UVSCIL) technique for photonic crystals patterning in LED manufacturing," Microelectronic Engineering, vol. 87, no. 5-8, pp. 963-967, May 2010.

[21] T. Müller, R. Kumpe, H. A. Gerber, R. Schmolke, F. Passek, and P. Wagner, "Techniques for analysing nanotopography on polished silicon wafers," Microelectronic Engineering, vol. 56, no. 1-2, pp. 123-127, May 2001.

[22] M. Colburn, B. Choi, S. Sreenivasan, R. Bonnecaze, and C. Willson, "Ramifications of lubrication theory on imprint lithography," MICROELECTRONIC ENGINEERING, vol. 75, no. 3, pp. 321-329, Sep. 2004.

[23] S. Reddy, P. Schunk, and R. Bonnecaze, "Dynamics of low capillary number interfaces moving through sharp features," PHYSICS OF FLUIDS, vol. 17, no. 12, Dec. 2005.

[24] S. Reddy and R. T. Bonnecaze, "Simulation of Fluid Flow in the Step and Flash Imprint Lithography Process," Microelectron. Eng., vol. 82, no. 1, pp. 60-70, Sep. 2005.

[25] S. Chauhan, F. Palmieri, R. T. Bonnecaze, and C. G. Willson, "Pinning at template feature edges for step and flash imprint lithography," Journal of Applied Physics, vol. 106, no. 3, p. $034902,2009$.

[26] S. Chauhan, F. Palmieri, R. T. Bonnecaze, and C. G. Willson, "Feature filling modeling for step and flash imprint lithography," Journal of Vacuum Science \& Technology B, vol. 27, no. 4, pp. 1926-1932, Jul. 2009.

[27] X. Liang, H. Tan, Z. Fu, and S. Chou, "Air bubble formation and dissolution in dispensing nanoimprint lithography," NANOTECHNOLOGY, vol. 18, no. 2, Jan. 2007.

[28] S. N. Reznik and A. L. Yarin, "Spreading of a viscous drop due to gravity and capillarity on a horizontal or an inclined dry wall," Phys. Fluids, vol. 14, no. 1, p. 118, 2002.

[29] B. Steinhaus, P. T. Spicer, and A. Q. Shen, "Droplet Size Effects on Film Drainage between Droplet and Substrate," Langmuir, vol. 22, no. 12, pp. 5308-5313, Jun. 2006. 
[30] J. GEORGES, S. MILLOT, J. LOUBET, and A. TONCK, "DRAINAGE OF THIN LIQUID-FILMS BETWEEN RELATIVELY SMOOTH SURFACES," JOURNAL OF CHEMICAL PHYSICS, vol. 98, no. 9, pp. 7345-7360, May 1993.

[31] L. Bureau and A. Arvengas, "Drainage of a nanoconfined simple fluid: Rate effects on squeeze-out dynamics," Phys. Rev. E, vol. 78, no. 6, p. 061501, Dec. 2008.

[32] M. Sellier and S. Panda, "Beating capillarity in thin film flows," INTERNATIONAL JOURNAL FOR NUMERICAL METHODS IN FLUIDS, vol. 63, no. 4, pp. 431-448, Jun. 2010.

[33] N. Alleborn and H. Raszillier, "Local perturbation of thin film flow," ARCHIVE OF APPLIED MECHANICS, vol. 73, no. 9-10, pp. 734-751, Apr. 2004.

[34] Y. D. Shikhmurzaev, Capillary Flows with Forming Interfaces. Chapman \& Hall/CRC, 2007.

[35] Y. D. Shikhmurzaev, "Singularities at the moving contact line. Mathematical, physical and computational aspects," Physica D: Nonlinear Phenomena, vol. 217, no. 2, pp. 121-133, 2006.

[36] J. Flitton and J. King, "Moving-boundary and fixed-domain problems for a sixth-order thinfilm equation," EUROPEAN JOURNAL OF APPLIED MATHEMATICS, vol. 15, pp. 713754, Dec. 2004.

[37] B. Fornberg, A Practical Guide to Pseudospectral Methods. Cambridge University Press, 1998. 


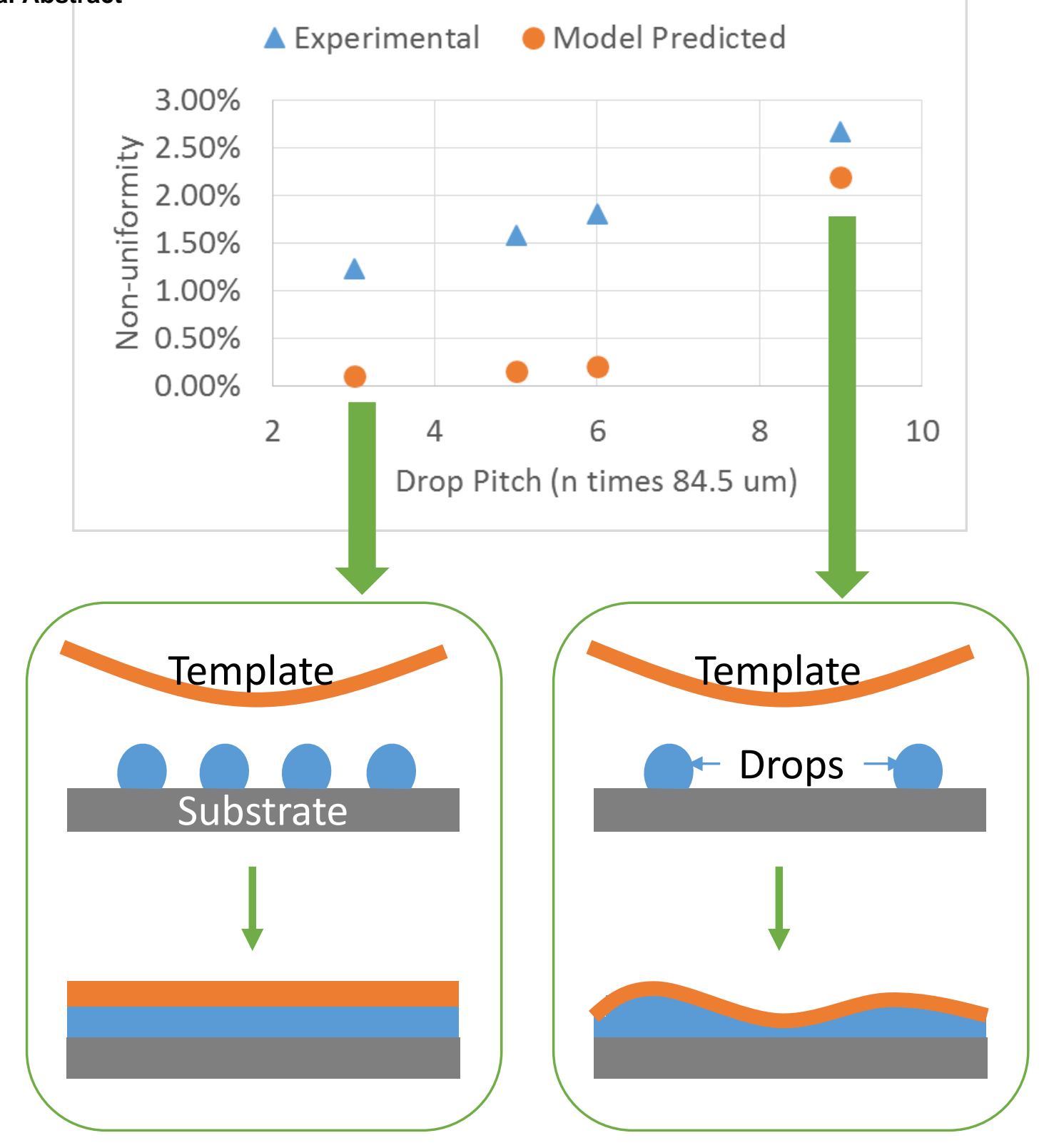

Inkjet drop placement affects residual layer thickness uniformity in Jet-and-Flash Imprint Lithography. 